\title{
INITAL PRESENTATION AND REFERRAL OF DIABETES MELLITUS
}

\author{
L. Nickerson ${ }^{1}$, R. Jayaraman ${ }^{2}$ \\ ${ }^{1}$ Paediatrics, ${ }^{2}$ University Hospital of Wales, Cardiff, UK
}

Background: NICE recommended in 2004 that children with suspected type 1 diabetes mellitus should be offered same day referral to a paediatric diabetic multidisciplinary team. On initial presentation of Diabetes Mellitus screening bloods are recommended. Current practise in a Welsh DGH was compared to these guidelines.

Methodology: A retrospective analysis of 71 patients in our unit over a ten year period (2000-10) was carried out. Case notes were analysed.

Results: Most presentations were in winter and autumn months. Polyuria (85\%), polydipsia (92\%), weight $\operatorname{loss}(62 \%)$, lethargy(35\%) were the main presenting symptoms. 20/71(28\%) presented in DKA. 54/71 (76\%) presented to general practitioner(GP). 17/71(34\%) presented to Accident \& Emergency. 13/54 (24\%) who presented to GP were referred to paediatric team more than 24 hours of initial presentation. Of these 2

patients were booked for blood tests, 8 patients were not diagnosed and no action was taken for 3 patients. The mean $\mathrm{HbA} 1 \mathrm{c}$ of these 13 patients was $11.4 \mathrm{mmol} / \mathrm{L}$ compared

to $10.4 \mathrm{mmol} / \mathrm{L}$ for the rest. $30 \%$ of these patients presented in DKA out of which $66 \%$ were severe DKA $(\mathrm{pH}<7.1)$. All the Intensive Care Unit admissions were delayed referrals. Children who were referred late also had more days of hospital admission.

Conclusions: Children who were referred more than 24 hours after initial presentation had higher HbAlc levels, were more likely to present in severe DKA, were hospitalised longer and had more likelihood of ITU admission. This analysis reiterates the importance of early recognition and referral of diabetes mellitus. 